\title{
Invited: Advanced neutral alkali beam diagnostics for applications in fusion research $^{\mathrm{a})}$
}

S. Zoletnik, ${ }^{1}$ G. Anda, ${ }^{1}$ M. Aradi, ${ }^{2}$ O. Asztalos, ${ }^{3}$ S. Bató, ${ }^{1}$ A. Bencze, ${ }^{1}$ M. Berta, ${ }^{4}$ G. Demeter, ${ }^{1}$ D. Dunai, ${ }^{1}$ P. Hacek, ${ }^{5}$ S. Hegedüs, ${ }^{1}$ G.H. Hu, ${ }^{6}$ T. Krizsanóczi, ${ }^{1}$ M. Lampert, ${ }^{1}$ D. Nagy, ${ }^{1}$ J. Németh, ${ }^{1}$ M. Otte, ${ }^{7}$ G. Petravich, ${ }^{1}$ G. I. Pokol, ${ }^{3}$ D. Réfy, ${ }^{1}$ B. Tál, ${ }^{1}$ M. Vécsei, ${ }^{1}$ and W7-X Team ${ }^{b}$

1) Wigner Research Center for Physics, Budapest, Hungary

${ }^{2)}$ Graz University of Technology, Graz, Austria

3) Budapest University of Technology and Economnics, Budapest, Hungary

${ }^{4)}$ Széchenyi University, Györ, Hungary

5) Institute for Plasma Physics, Czech Academy of Sciences, Prague, Czech Republic

${ }^{6)}$ Institute for Plasma Physics, Chinese Academy of Sciences, Hefei, China

7) Max Planck Institute for Plasma Physics, Greifswald, Germany

Diagnosing the density profile at the edge of high temperature fusion plasmas by accelerated Lithium beam is a known technique since decades. By knowledge of the relevant atomic physics rate coefficients the plasma electron density profile can be calculated from the relatively calibrated light profile along the beam. Several additional possibilities have already been demonstrated: CXRS for ion temperature/flow, Zeeman polarimetry for edge plasma current; therefore the Li-beam diagnostic offers a wealth of information at the plasma edge. The weaknesses of the method are the relatively faint light signal, background light and technical difficulties of the beam injector which usually seriously limit the applicability. In this talk we present systematic developments in alkali-beam diagnostics ( $\mathrm{Li}, \mathrm{Na}$ ) both for the injector, observation system and detectors which resulted in strongly increased capabilities. Advanced systems have been built and microsecond scale density profile, turbulence and zonal flow measurement demonstrated. A novel edge current measurement technique has also been designed and components tested with potentially microsecond-scale time resolution. Additional possibilities of these advanced systems for spectral measurements (CXRS, various Zeeman schemes) are also discussed.

\section{INTRODUCTION}

Diagnosing magnetically confined fusion plasmas with neutral Lithium beams have been proposed and demonstrated as early as the 1970 's ${ }^{1-3}$. The first and most important application was Beam Emission Spectroscopy (BES), the determination of the edge density profile via observation of the beam light emission. The choice of Lithium is the result of a relatively simple Hydrogen-like electron configuration, low sensitivity of excitation and ionization cross-sections to plasma electron temperature and well measurable visible line $(670.8 \mathrm{~nm})$ emission from the first excited state. Also, alkali ion sources are easy to realize technologically as heated ceramic materials are well known as alkali ion emitters. Besides BES it has also been proposed and demonstrated to use Lithium beams for determination of the magnetic field direction through measurement of the Zeeman split spectrum of the emission line ${ }^{4-6}$ and as a source of electrons for charge exchange measurement of impurity ions ${ }^{7-9}$. These measurement techniques and the results obtained are summarized in a review paper ${ }^{10}$.

\footnotetext{
a) Invited paper published as part of the Proceedings of the 22nd Topical Conference on High-Temperature Plasma Diagnostics, San Diego, California, April, 2018.

b) ) See R. C. Wolf et al., Major results from the first plasma campaign of the Wendelstein 7-X stellarator, Nucl. Fusion 57, 102020 (2017).

E-mail of contact author: zoletnik.sandor@wigner.mta.hu
}

Although all the above measurement possibilities have been demonstrated the routine application of the technique was limited to the measurement of the density profile with at most millisecond time resolution. The reason is the limited light intensity stemming from both the 1-2 mA ion equivalent beam current, low light detection efficiency of fibre-photomultiplier systems and disturbances from the fluctuating background light. However, turbulence and transient phenomena during the L$\mathrm{H}$ transition and ELMs call for reliable measurements on sub-millisecond timescale. Some better time resolution density profile measurements could be achieved with the conventional Li-BES systems using careful correction for the background changes ${ }^{11}$ during ELMs. Edge turbulence measurement results were also done through correlation functions ${ }^{12,13}$ and power spectra ${ }^{14}$. In the ScrapeOff Layer (SOL) the higher fluctuation amplitude also enabled more detailed studies of blob dynamics ${ }^{15}$. From these results it became obvious that Li-beam diagnostic could offer more measurement possibilties if the detected light intensity could be substantially increased.

This paper describes consistent efforts in order to improve both beam performance on the one hand and optical and detection efficiency on the other hand. These developments enabled to increase the time resolution of the density profile measurement by alkali beam diagnostic into the microsecond range while also providing twodimensional information. The paper is organized as follows. Sections II and III describe improvements to the beam injector and beam control, respectively. Section IV gives an overview of various observation system and 
detector implementations. Additional measurement possibilities are discussed in Section V and the Conclusion gives a brief overview of achievements.

\section{ADVANCED ALKALI BEAM INJECTOR}

The ion source of diagnostic Li-beam injectors use some kind of Lithium aluminosilicate material, typically Eucryptite $\mathrm{LiAlSiO}_{4}$. Heated to $1300{ }^{\circ} \mathrm{C}$ temperature Lithium ions become mobile and an electric field can extract them from the surface. The extracted current density is determined by the temperature of the material or, at high enough temperature, by the space charge limit set by the acceleration geometry and voltage. In order to keep the material in place and replace the extracted charge the eucryptite material is embedded in a porous Tungsten or Molybdenum matrix. In the original ASDEX ion source ${ }^{16}$ a $14 \mathrm{~mm}$ diameter source, manufactured by Heatware Corp., is used. In this setup heating is provided by $\approx 30 \mathrm{~A} / 6 \mathrm{~V}$ AC current flowing through a Molybdenum filament embedded in an Alumina filling. It was found that the reliably reachable temperature is about $1300{ }^{\circ} \mathrm{C}$ where $\approx 3 \mathrm{~mA} \mathrm{Li}$ ion current can be extracted, limited by the ion source. Further increasing the temperature increased the extracted current but seriously limited the lifetime of the heating filament. Although various filling materials were tried no solution was found, therefore the heating element was replaced by a specially manufactured Silicon Carbide disc. By appropriately selected Carbon content and heat treatment this heater can be operated at much higher temperatures. With appropriate heat shields and $\approx 80 \mathrm{~A} / 4 \mathrm{~V}$ AC heating $1380{ }^{\circ} \mathrm{C}$ can easily be reached. Due to special composition of the emission material and proper heat treatment the extracted ion current reaches $5 \mathrm{~mA}$, corresponding to about $3 \mathrm{~mA} / \mathrm{cm}^{2}$ current density. This value is consistent with data from the literature ${ }^{17}$ where ion sources operating below $1300{ }^{\circ} \mathrm{C}$ are reported to deliver up to $1.5 \mathrm{~mA} / \mathrm{cm}^{2}$ current density. The total extractable charge was tested by operating an ion source at a moderate $2 \mathrm{~mA}$ ion current continuosly for several hours per day. The final extracted charge was about $20 \mathrm{mAh}$ what is about $30 \%$ of the Lithium content in the emission material. This new type of ion source is licensed for manufacturing to Adimtech Kft.

It has to be noted that an alternative Lithium ion source and beam accelerator was developed for DIII-D ${ }^{18}$ and JT-60U ${ }^{19}$ where a large diameter $(5 \mathrm{~cm})$ source is heated with an electron beam and up to $10 \mathrm{~mA}$ ion current is reported with lower ion current density. This solution we found less compact and technically more challenging in the environment of a fusion experiment.

Ion acceleration is done using a slighly modified version of the original ASDEX geometry ${ }^{16}$. At $60 \mathrm{kV}$ beam energy the space charge limit for the ion extraction is about $5 \mathrm{~mA}$ from a $14 \mathrm{~mm}$ diameter Lithium ion source, therefore the full emission capacity of the new ion source

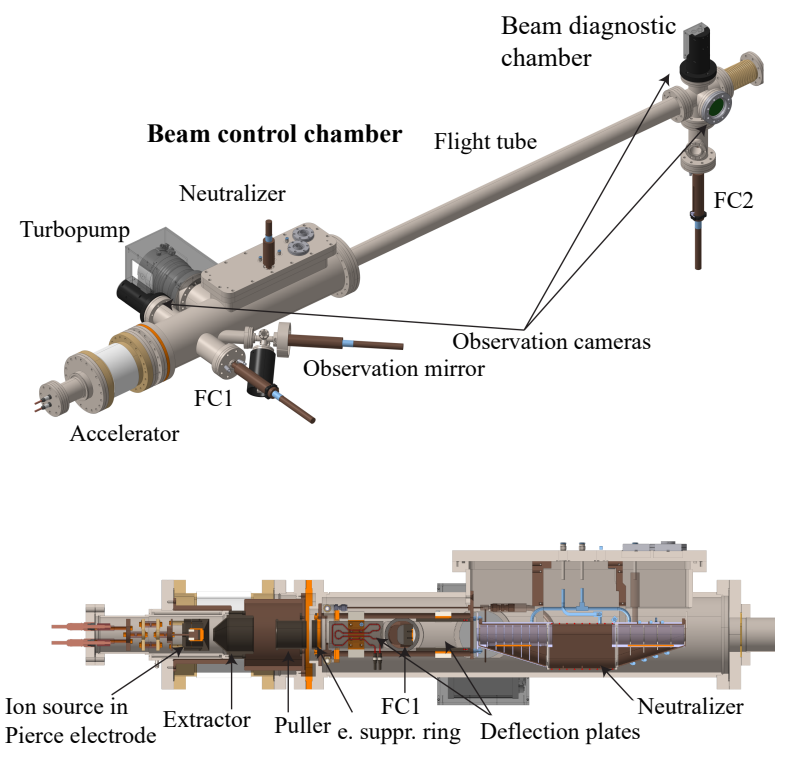

FIG. 1. Engineering design and cross-section of the beamline.

can be used. With a somewhat modified geometry up to $8 \mathrm{~mA}$ ion current can be extracted from a $19 \mathrm{~mm}$ diameter source. However, focusing the full extracted beam to small diameter was found to be difficult. On large fusion devices the typical distance from the ion source to the plasma is around $4-5 \mathrm{~m}^{19-22}$. The beam FWHM at this distance could be reduced to $2.5-3.5 \mathrm{~cm}$ for the small and large diameter source, respectively. The measured ion equivalent neutral beam current at this distance is typically not more than $30-50 \%$ of the extracted ion current. The rest of the beam can be found in a broad halo. This behavior is not fully understood. It is known that electrons play a role in neutralizing the space charge of the ion beam ${ }^{23}$ therefore it is suspected that cumulative space charge effect of the electron cloud and the ion beam between the accelerator and the neutralizer modifies the ion velocity in the outer regions of the beam and generates the halo.

The next element down the beamline in Fig. 1 is the neutralizer which is traditionally implemented ${ }^{3}$ as a Sodium oven filling a tube around the beam with Sodium vapor. Using the neutralizer for longer pulses contaminates components, causes sparks in the accelerator and distorts the beam shape by charging of non-conducting contaminated surfaces. For long-pulse operation a modified neutralizer was developed ${ }^{24}$. The basic observation is that at the pressure needed for beam neutralization a few $\mathrm{cm}$ from the neutralizer the Sodium vapor flow becomes molecular and the outflow can be limited by diaphragms. The new recirculating neutralizer (see Fig. 1) has a central Sodium vapor filled cell with two $2.5 \mathrm{~cm}$ diameter holes for the beam. The cell is kept at $250-280{ }^{\circ} \mathrm{C}$ temperature where the Sodium does not condense on the walls but kept in liquid state at the bottom. On the two sides multiple diaphragms are installed in cones and kept at $\approx 130{ }^{\circ} \mathrm{C}$ where Sodium condenses and flows back into 
the central cell. The relative sizes of the central cell and the cones were determined ${ }^{24}$ in order to minimize Sodium loss within a certain full neutralizer length. The central cell has $1 \mathrm{~kW}$ electric heating and pressurized air cooling, while the cones have only cooling as heat conduction is sufficiently high from the central cell. Between plasma measurements the central cell is kept at a standby temperature of $\approx 180{ }^{\circ} \mathrm{C}$ where the Sodium pressure is two orders of magnitude lower than during operation. Before beam operation the neutralizer is heated up in about one minute. To prevent any Sodium contamination during this period pneumatically operated shutter plates are covering the entrance and exit diaphragms. After beam operation pressurized air cools down the neutralizer cell to standby temperature.

Between the accelerator and the neutralizer two deflection plate pairs are installed which deflect the ion beam in horizontal and vertical direction. A pneutmatically movable Faraday cup (FC1) and a mirror for ion source observation can be moved into the beamline between them. The Faraday cup can measure the ion current and also observe the beam shape with a camera ${ }^{23,24}$. The deflection plates are heatable in order to occasionally clean them from any Sodium deposits. During cleaning a miniature pneumatic shutter protects the accelerator vessel from Sodium contamination.

At the place of the injector typically $1-10 \mathrm{mT}$ stray magnetic field is present at the fusion devices, which would seriously affect beam position and shape. To prevent this massive magnetic shielding is needed and installed $^{16,18,25}$. In our new beam design the whole vacuum chamber containing the neutralizer and the deflection plates is manufactured from Nickel plated soft iron, therefore no additional shielding is required in this region. In the accerator the holder of the extractor electrode and the electrode itself is manufactured from soft iron and a shielding tube is installed around the puller electrode, thus providing integrated shielding. The extractor holder strucure and the ion source heating current feedthroughs have internal water cooling channels, therefore all structures, except the ion source and the Pierce electrode, are kept cold.

The new Li-beam injector is a compact device requiring only a single turbomolecular pump. All high voltage and control elements are removed from the beam and installed at the laboratory, therefore protecting them from neutron caused disturbancies which otherwise happened close to the fusion device. Only a compact high voltage transformer for the ion source heating, protection and load resistors and some fibre communication units are located at the beam. Usually a separate beam diagnostic chamber is installed along the beamline close to the fusion device port. Here a Faraday cup and two video cameras enable characterization of the beam shape, movement, current and neutralization efficiency. A detailed description of the injector is published in Ref. ${ }^{24}$.

Although the beam is designed for $60 \mathrm{kV}$ operation, but with some improvements to the high voltage trans-

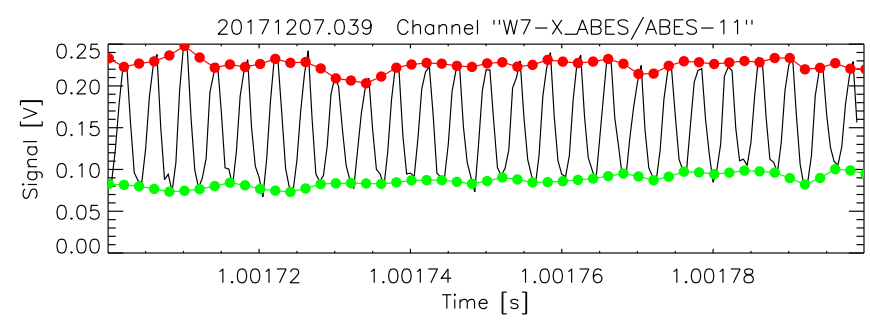

FIG. 2. Fast beam chopping measuremeent example in the Wendelstein 7-X alkali beam diagnostic. The black curve is the measured APD signal in one observation channel. The green curve is the extracted background, while the red the background+beam signal.

former and cabling it has recently been tested up to 100 $\mathrm{kV}$. Besides Lithium, Sodium and Rubidium ion sources have also been built and tested.

\section{ADVANCED BEAM CONTROL AND CHOPPING}

For background light measurement the beam must be chopped, that is switched on/off. For this purpose high enough voltage is applied (500-1000 V) between deflection plates which moves the beam completely out of the observation volume. Theoretically the maximum chopping frequency is limited to a few $\mathrm{MHz}$ by the flight time of the ions through the deflection plates. In practice high-voltage (HV) switches and charging-discharging the capacitance represented by the deflection plates and the connecting cables impose limitations on the frequency and/or the duration of the operation. On W7-AS ${ }^{26}$ beam chopping and hopping (few cm movement) was demonstrated up to $250 \mathrm{kHz}$ and $400 \mathrm{kHz}$, respectively. An alternative scheme was developed by the ASDEX Upgrade team ${ }^{25}$ by switching the voltage between the ion source and the extractor electrode. This is more efficient for beam extinction but due to the higher voltage its frequency is limited to $2 \mathrm{kHz}$. In our upgraded deflection chopping system ${ }^{24}$ Behlke HTS 31-03-GSM-HFS HV switches connect $1 \mathrm{kV}$ with opposite polarity on one pair of deflection plates. This improved beam extinction while keeping the maximum frequency at about 250 $\mathrm{kHz}$. In this system fast $\mathrm{HV}$ switches are installed on both deflection plate pairs, therefore the beam can be chopped/hopped in any direction.

Calculating the background and beam light with slow chopping is straightforward, but the two light signals are shifted in time. For fast changing background, e.g. during ELMs chopping in the $100 \mathrm{kHz}$ range is necessary. At several $100 \mathrm{kHz}$ frequency the switchover time of the measured beam light signals is determined by the bandwidth of the signal amplifiers and the exact time of the switch is also shifted few $100 \mathrm{~ns}$ along the beam due to finite velocity of the atoms. A self-calibrating demodulation technique has been developed ${ }^{22}$ for this purpose where the exact temporal change of the detected signal 
is extracted from the average of several chopping periods and/or extrapolation from other channels along the beam. This beam modulation signal is used for iteratively demodulating the beam signal and determining the background as shown in Fig. 2.

\section{IMPROVED OPTICAL AND DETECTOR SOLUTIONS}

Traditionally the observation system of Li-BES diagnostics for density profile and turbulence measurements consists of an optical head and fibre bundles leading light to individual PhotoMultiplier (PM) detectors ${ }^{14,16,18}$. The peak detected light intensity in these systems is in the range of $10^{6}-10^{8}$ photon/s which, at $1 \mathrm{MHz}$ bandwidth, results in $\geq 10 \%$ photon statistical noise at the peak of the profile, while 1-2\% noise would be desirable for fast measurements. After exploring recent capabilities of Avalance PhotoDiode (APD) detectors ${ }^{27}$ it was found that, due to their higher Quantum Efficiency (QE), above $10^{9} \mathrm{ph} / \mathrm{s}$ their overall noise is about a factor of two lower than that of PMs. Additionally they are more compact and not sensitive to magnetic field therefore can be placed close to the fusion device and fibre bundles can be avoided. Therefore the design aim of advance systems was to increase the light collection solid angle to the point where $\approx 10^{10} \mathrm{ph} / \mathrm{s}$ peak light intensity can be reached and APD detectors can be used.

Fig. 3. shows the setup of the TEXTOR Li-BES observation system we built using this principle. An in-vessel optics is used for increasing the observation solid angle to $\approx 6 \cdot 10^{-4} \Omega / 4 \pi$ and avoiding the direct view on the limiter below. About 10 percent of the light is coupled to a CCD camera for beam monitoring but most of the light falls on an APD matrix array. Both optical branches use a $2 \mathrm{~nm}$ bandwidth interference filter at a location where light rays are parallel to better than 5 degrees. The APD detectors have $5 \times 5 \mathrm{~mm}$ sensitive area (Hamamatsu S8665$55)$ and over $85 \% \mathrm{QE}$ at $670 \mathrm{~nm}$. The magnification of the optics to the APD detectors is 1 but to maximize light intensity a $1 \times 3 \mathrm{~cm}$ rectangular lens is mounted in front of each detector with the detector in the focal point. This effectively increases the measurement range of one detector to a $1 \times 3 \mathrm{~cm}$ (radial $\times$ toroidal) area on the beam, thus reducing sensitivity to toroidal beam movement and width changes. The APDs are mounted on a temperature controlled Copper tab to stabilize their gain. Although this $35 \mathrm{kV}$ Li-beam had only $1 \mathrm{~mA}$ ion equivalent current this observation system yielded $4 \cdot 10^{10} \mathrm{ph} / \mathrm{s}$ peak detected light intensity and peak $\mathrm{SNR} \approx 60$, which was enough for edge plasma turbulence, zonal flow and few microsecond scale density profile measurements ${ }^{28}$.

The TEXTOR setup also enabled extension of the diagnostic to quasi-2D resolution by hopping the beam updown a few $\mathrm{cm}$. This effecively moves the measurement volume poloidally. $400 \mathrm{kHz}$ hopping frequency is above the bandwidth of edge turbulence and thus, with 1-2 cm

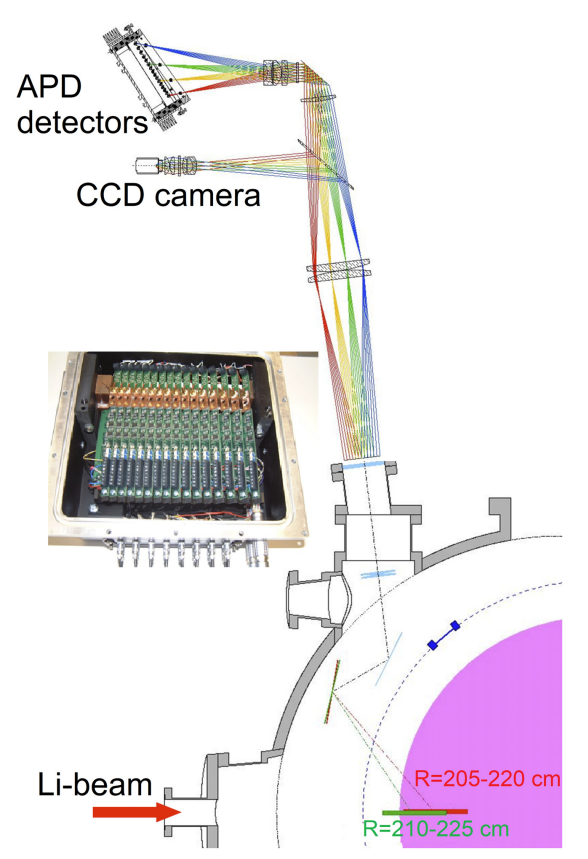

FIG. 3. Design of the optics for the TEXTOR Li-BES diagnostic and photograph of the APD detector array.

beam movement, the wavelength and propagation velocity of edge turbulence can be measured ${ }^{28}$.

On the COMPASS tokamak ${ }^{29}$ a similar optical setup was installed ${ }^{30}$ with 18 APD detectors looking at the beam perpendicularly. Due to the advanced injector and larger solid angle obseravation system the light intensity is even higher than on TEXTOR enabling measurement of density profile changes on microsecond timescale ${ }^{31}$.

\section{A. Two-dimensional resolution}

Although beam hopping enabled gaining some information on the poloidal strucure of turbulence it limits the frequency range of the measurement and interferes with beam chopping. Therefore it would be desirable to obtain real two-dimensional (radial-poloidal) resolution by looking at the Li-beam from B-parallel direction. Such a view is implemented on $\mathrm{JET}^{32}$ albeit with limited spatial coverage and light collection efficiency. For EAST we designed and built an optimized 2D Li-BES system ${ }^{22}$ capable of 2D resolution (Fig. 4). Due to space restrictions the observation system had to be located in the same large equatorial port where the beam is injected. After extensive modeling using the 3D BES code RENATE ${ }^{33}$ a compromise was found between sufficient radial and poloidal resolution and enough Doppler shift of the beam light on this device with regular Lithium wall conditioning. The setup of the full Li-BES diagnostic on EAST is shown in Fig. 4. Light is collected by an $80 \mathrm{~mm}$ diameter first lens located at about $60 \mathrm{~cm}$ from the measure- 
(b)

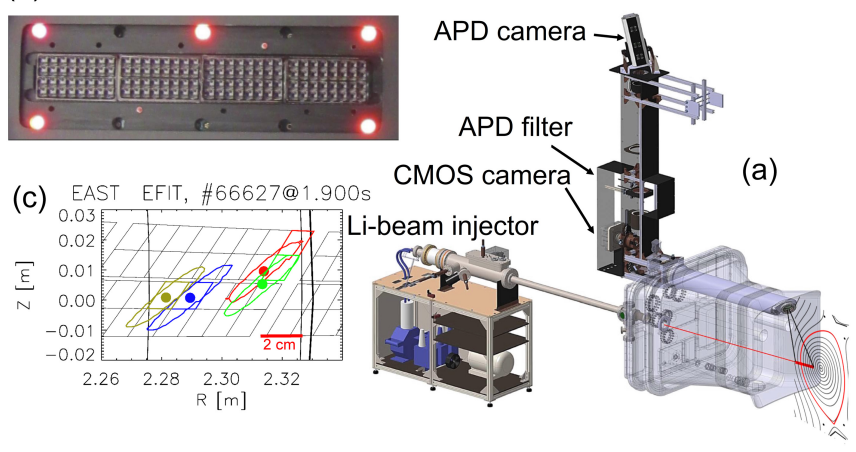

FIG. 4. (a) Engineering design of the EAST Li-BES system with typical EFIT flux surface contours; (b) photograph of the $4 \times 32$ pixel APD matrix; (c) calculated mapping of APD pixels on the vertical beam plane (black rectangles), some effective $50 \%$ sensitivity curves and their centroids (coloired curves and dots).

ment volume. $\left(\Omega / 4 \pi \approx 1.1 \cdot 10^{-3}\right)$ When not used the window is protected by a shutter. Light is transmitted through an imaging system to a two-dimensional (4x32 pixel) APD detector matrix camera (Fusion Instruments APDCAM-10G-4x32) equipped with microlens array. A few percent of the light is directed to a CMOS camera (PhotonFocus MV1-D1312(IE/C)-G2) which provides an overview image of beam quality and enables spatial calibration of the system. Both detectors transmit data directly to computer memory therefore measurement time is only limited by computer memory. The APD signals are sampled typically at $2 \mathrm{MHz} / 14 \mathrm{bit}$. This direct imaging approach results in typically $1 \cdot 10^{10} \mathrm{ph} / \mathrm{s}$ peak beam light intensity which means up to $\mathrm{SNR}=60$ on $500 \mathrm{kHz}$ analog bandwidth.

The effective sensitivity distribution of the APD pixels on the radial-poloidal plane of the tokamak is determined by the intersection of the beam and the observation line of sight projected onto the radial-poloidal plane. Due to the oblique observation the effective sensitivy areas are inclined and extend to $2-3 \mathrm{~cm}$ both radially and horizontally as shown in Fig. 4(c). This is taken into account and to some extent corrected for in the density profile calculation.

Filtering the 1.4-2.5 nm Doppler shifted Li-beam light was a specific problem as factor of 100 higher radiation is expected at the unshifted $670.8 \mathrm{~nm}$ Lithium line from the plasma edge. In order to keep the filter cutoff sufficiently sharp the light ray angles on the filter had to be limited to 3 degrees which resulted in a $165 \mathrm{~mm}$ diameter filter size. To compensate for temperature changes and manufacturing tolerances a special filter holder was designed and manufactured which can uniformly heat this large diameter filter up to $60{ }^{\circ} \mathrm{C}$. This setup enables effective suppression of the background light but its amplitude can still reach $100 \%$ of the peak beam light intensity, especially during ELMs. This can be removed by the fast beam chopping technique described above. Using an

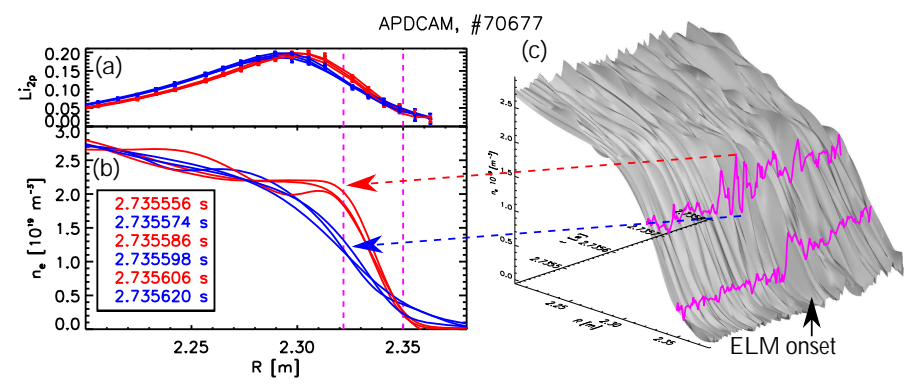

FIG. 5. Density profile measured around the onset of a small ELM in EAST with $4 \mu s$ time resolution. The background corrected light (a) and density (b) profiles are at extrema of the precursor oscillation seen in the time evolution of the density profile in (c).

implementation of a Bayesian calculation algrorithm ${ }^{34}$ the edge plasma density profile can be calculated with few microsecond time resolution as illustrated in Fig. 5. At the same time the poloidal flow velocity and poloidal wavelength of modes can also be determined albeit with only ms time resolution ${ }^{22}$.

\section{B. Improved spatial resolution with Sodium beam diagnostic}

One limitation of the Li-beam diagnostic is the relatively long (27 ns) lifetime of the $\mathrm{Li}_{2 p}$ excited state, which multiplied by the $10^{6} \mathrm{~m} / \mathrm{s}$ beam velocity, means $2-3 \mathrm{~cm}$ smearing along the beam path. Although collisional deexcitation reduces this length somewhat it still poses a limitation in spatial resolution. In case of Sodium the excited state lifetime is shorter and the spatial smearing is reduced to about $1 \mathrm{~cm}$. The possibiliy of a Sodium beam diagnostic and density calculation was demonstrated on ASDEX Upgrade ${ }^{35}$. On the Wendelstein 7-X (W7-X) stellarator we have recently installed a Sodium beam diagnostic for the measurement of the density profile and turbulence through the O-point of the edge island at the "bean-shaped" cross section where the density profile is expected to be steep (Fig. 6(a)). The ion source is the same construction as the Lithium one, just Lithium is replaced by Sodium and lower heating temperature is sufficient. The max. $60 \mathrm{kV}$ Sodium beam is injected horizontally in the equatorial plane of the stellarator while observation is done perpendicularly, from an upper port. The first lens collects all light through a $182 \mathrm{~mm}$ diameter window about $2.5 \mathrm{~m}$ above the beam $\left(\Omega / 4 \pi \approx 3 \cdot 10^{-4}\right)$ where no protection or cooling was necessary. A closer window would have to be smaller due to active cooling, shutter and microwave protection requirements, resulting in the same collection angle. After the window the light passes through a $236 \mathrm{~mm}$ diameter 3 cavity filter and focused onto a fibre plate where it is coupled to 40 pc. $0.6 \times 5 \mathrm{~mm}$ fibre bundles built from $50 \mu \mathrm{m}$ diameter $\mathrm{NA}=0.55$ fibres. Each fibre bundle collects light from an 
$\approx 0.5 \times 4 \mathrm{~cm}$ (radial $\times$ toroidal $)$ area in order to inte grate across the whole beam toroidally. At the other en of the $2 \mathrm{~m}$ long bundles the fibres are arranged into a : $\mathrm{mm}$ diameter circle and coupled to a special APDCAM 10G-FC64 device which contains 64 pc. $3 \mathrm{~mm}$ diame ter APD and MultiPixel Photon Counter (MPPC) detec tors (Hamamatsu S8664-30K and S13360-3075CS). Th MPPC detectors are installed for the SOL measurement where the light level is expected to be below $10^{9} \mathrm{ph} / \mathrm{s}$ an these new high-gain devices provide better $\mathrm{SNR}^{36}$. Sim ilarly to the EAST Li-BES setup a few percent light i coupled to a CMOS camera for beam quality check anı spatial calibration.

An example CMOS image with the measured contour of fibre bundle $50 \%$ sensitivity areas overlaid is shown in Fig. 6 from the first measurements at the end of the OP 1.2a W7-X campaign At the plasma edge a bright stripe can be observed, which is caused by the influx of thermal Sodium from the beam neutralizer. Although even this high background can be removed with the fast beam chopping technique the neutralizer vapor will be changed to another alkali metal to get rid of the disturbance. Deeper in the plasma the peak beam signal is about $1 \cdot 10^{10} \mathrm{ph} / \mathrm{s}$ in agreement with RENATE modeling. A beam light profile from a $20 \mu s$ long time interval, corrected for background and thermal Sodium signal with fast chopping, is shown in Fig 6(c). The light profile can be fit within error bars by the Bayesian density calculation algorithm (Fig 6(d)) and a maximum in the density profile appears within $1 \mathrm{~cm}$ of the $\mathrm{O}$-point of the island in the vacuum magnetic field calculation. Due to the high time resolution of the system blob-like structures can also be seen in the island region, inside and outside it. In the island region they show much more complex dynamics than seen in tokamaks. This behaviour will be investigated in detail also using the quasi 2D resolution through poloidal beam hopping in the next operation campaign.

\section{Radiation effects}

In the above described diagnostic systems the detectors are located close to the fusion device thus neutrons and gamma photons cause noise in the signals. They appear as individual pulses with a temporal width set by the inverse of the signal amplifier bandwidth. Such pulses are observed only during NBI heating. At present it is not clear whether they are caused directly by neutrons or prompt gammas generated by them. An algorithm has been developed to find the pulses by locating high positive derivate closely followed by a negative one. The threshold for the derivative is a free parameter which is set to a value where minimum number of pulses are found without NBI heating. Fig. 7 shows the number of detected pulses as a function of limit. In this case the derivative limit is set to 0.03 . In the NBI phase the pulses below this limit are indistinguishable from noise and they only cause a negligible increase in
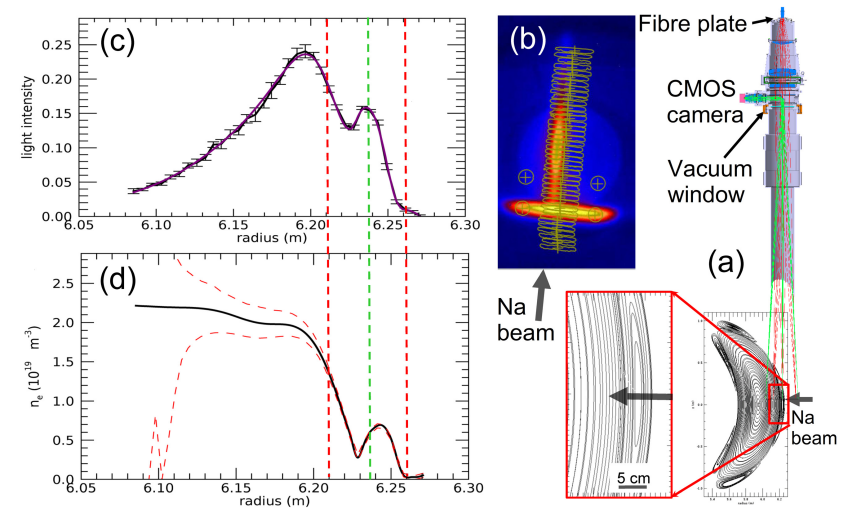

FIG. 6. (a) Geometry of the W7-X Sodium beam diagnostic; (b) False color CMOS camera image of Sodium beam injection into the W7-X plasma. The yellow contours are the measured $50 \%$ sensitivty limits of the optical channels. (c) and (d) are light profile and calculated density from data collected in a $20 \mu s$ timerange (20171207.051@1.6s), respectively. Red and gren dashed lines indicate the island separatrix and O-point in the vacuum field calculation, respectively.

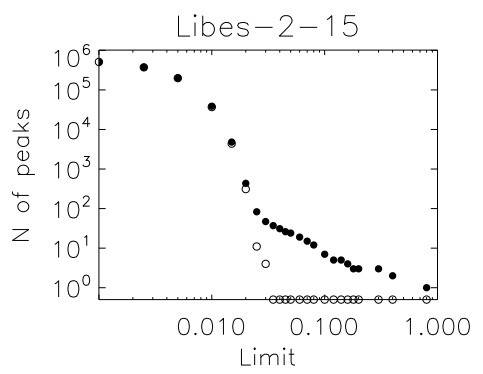

FIG. 7. Number of radiation induced pulses found in a $1 \mathrm{~s}$ long EAST Li-BES signal (shot 69426) as a function of limit. Filled symbols: with 1.5 MW NBI heating, open symbols: without NBI heating.

the noise level. The samples in the located pulses are substituted by linearly interpolated ones. On the TEXTOR, KSTAR, EAST, and MAST ${ }^{37}$ APD detector systems the number of affected samples remained well below $1 \%$, therefore after post-processing the radiation induced noise is negligible. Comparing discharges with different NBI power an approximately linear tendency was found indicating that the source is beam-plasma interaction. In measurements with an APDCAM camera on the ASDEX Upgrade FILD5 diagnostic ${ }^{38}$ during 20 MW NBI heating about $10 \%$ of the samples were affected indicating that at high power devices neutron shielding will be necessary. However, none of the APDCAM systems showed any sign of radiation damage during 3-4 years of operation. The CMOS camera also shows regular spots during NBI heating, but no damage was experienced after years of operation.

\section{ADDITIONAL MEASUREMENT POSSIBILITIES}

The advanced alkali beam diagnostics have 1-2 orders of magnitude higher light intensity than earlier systems, 
which resulted in greatly improved temporal resolution in the edge density profile and turbulence measurement. In this section we investigate whether an impovement could also be achieved in other known capabilities of the Lithium beam diagnostic.

\section{A. Edge current measurement}

Measuring the poloidal magnetic field, and through that the plasma current distribution, at the plasma edge would be interesting for testing ELM theories and especially the triggering condition of the instability.

\section{Zeeman split measurement}

A proven measurement technique for the edge current distribution with the Li-beam relies on observing changes in the Zeeman split spectral line structure in reponse to changes in the poloidal magnetic field. In magnetic field the spectrum consists of a central $\pi$ line group and two $\sigma$ line groups on its sides. The separation between these line groups depend on the magnetic field strength, while their polarization and relative amplitude depends on the magnetic field and observation direction. The seperation at a few Tesla magnetic field is only $\approx 0.1 \mathrm{~nm}$ which can be measured by a suitable spectrometer but diffucult to achieve good enough time resolution so as to study the role of edge current in different plasma phenomena. Diagnostics measuring the polarization of the $\sigma$ component separated by a narrow etalon filter have been installed on DIII-D ${ }^{4}$ and JT-60U ${ }^{5}$ and indeed the edge current changes in some cases even during the ELM cycle could be revealed ${ }^{39}$. On $\mathrm{JET}^{6}$ the method using the $\sigma / \pi$ intensity ratio has been demonstrated by careful analysis of spectrometer data from long integration times. Below we present first considerations on the possible use of these techniques on the EAST Li-BES diagnostic.

We believe the polarization method is unsuitable as the optical system in the long port of the tokamak is too complex to keep the polarization state of the emission. Concerning the line ratio method we performed modeling of the spectra collected through the existing $80 \mathrm{~mm}$ first lens, as shown in Fig. 8(a) for a moderate $40 \mathrm{keV}$ beam energy. Due to the strongly variing observation angle on the lens surface the lines are Doppler broadened and the components strongly overlap. Limiting the lens diameter to $20 \mathrm{~mm}$ (Fig. 8(b)) the overlap can be avoided. The transmission curves of an etalon from $\operatorname{Ref}^{5}$ shifted to two different wavelengths are overplot the spectra. Disregarding any detailed optical considerations we roughly assessed the measurement possibilities by multiplying the two transmission curves with the full spectrum and integrating in the plotted wavelength range to yield values $S_{1}$ and $S_{2}$. The relative change of $R=S_{1} / S_{2}$ in response to a poloidal field variation changing the field direction by 1 degree is plotted in Fig. 8(c) as a func-
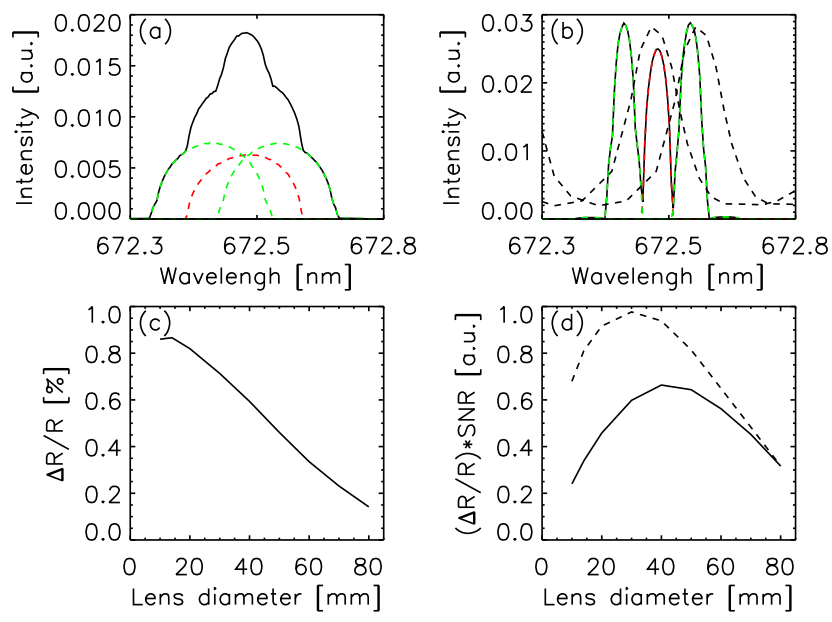

FIG. 8. (a) Modeled spectrum from $40 \mathrm{keV}$ beam through the existing $80 \mathrm{~mm}$ first lens in the EAST Li-BES diagnostic. The red dotted and green dashed curves show the $\pi$ and $\sigma$ components, respectively; (b) Spectrum through a $20 \mathrm{~mm}$ diameter window and transmission curves of an etalon (black dashed) based on $\operatorname{Ref}^{5}$; (c) Relative change $\Delta R / R$ for $1 \mathrm{deg}$ magnetic field direction change as a function of firs lens diameter; (d) $\Delta R / R$ normalized to signal noise for circular (solid line) and slit (dashed line) lenses.

tion of different lens radii. The Doppler broadening of the lines clearly reduces sensitivity already starting at $20 \mathrm{~mm}$ lens diameter. However, reducing the lens diameter also means reduction in the signal level, therefore the relavant quantity is $(\Delta R / R) \times S N R$ as plotted in Fig. 8(d). Here we assumed that the noise is in the photon statistics dominated regime using MPPC detectors. In this case the optimum lens diameter is shifted to 40 $\mathrm{mm}$. Some improvement can be achieved by using a rectagular lens aligned perpendicularly to the beam velocity to minimize Doppler broadening while maximizing signal as shown by the dashed line.

Assuming only 1\% thoughput in an optical setup with etalon compared to the present setup the detected photon flux would be $\approx 10^{8} \mathrm{ph} / \mathrm{s}$. On an MPPC detector with $100 \mathrm{kHz}$ bandwidth this would mean about $10 \%$ noise. This way in this prospective system to achieve $1 \mathrm{deg}$ statistical error the detector bandwith should be reduced to $1 \mathrm{kHz}$. This rough estimation indicates that measurement of the poloidal field using the Zeeman split is worth further exploring for the EAST Li-BES diagnostic.

\section{Atomic beam probe}

The magnetic field measurement through the Zeeman split requires special technology and has limited time resolution therefore an alternative method has been devised. When the high energy atoms in the beam are ionized the resulting ions travel on a Larmor trajectory toroidally deflected by the poloidal magnetic field. If the Larmor radius is large enough the ions can be collected outside 


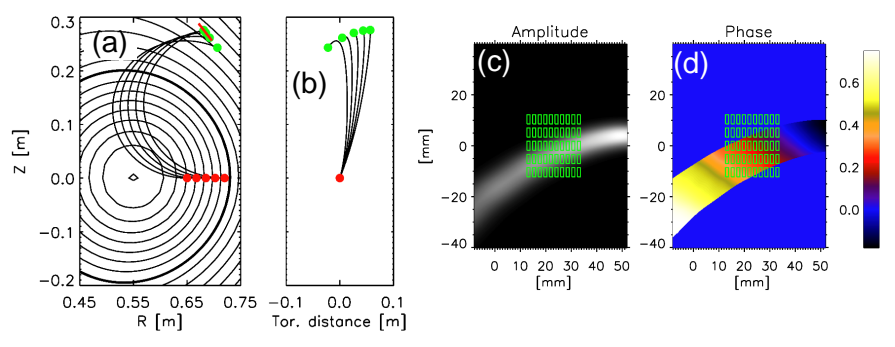

FIG. 9. Calculated $45 \mathrm{keV}$ Sodum ion trajectories in the COMPASS tokamak (discharge 15579, $B_{t}=1.15 T, I_{p}=$ $300 k A)$. (a) Toroidal view (left) and (b) radial view along the beam injection. The red dots are the starting points of the ions, the green points are arrival to the detector. (c) and (d) are modeled ion beam current and modulation phase (rad) distributions on the detector with outlines the 5x10 Faraday cups .

the plasma and from their toroidal deflection some information can be gained on the poloidal field. With Lithium ions the method is usable only in small devices ${ }^{40}$ and low magnetic fields but with heavier Alkali ions the edge region of medium-size fusion experiments can also be diagnosed ${ }^{41}$. As the toroidal deflection is in the few $\mathrm{cm}$ range the beam diameter should be limited to a few $\mathrm{mm}$ where the beam current is in the $100 \mu \mathrm{A}$ range. Assuming $\approx 10 \mathrm{~cm}$ beam penetration into the plasma still 1-10 $\mu A$ ion current is expected from a $1 \mathrm{~cm}$ region. This is well measurable with $\mathrm{MHz}$ bandwidth, therefore the technique has intrinsically high time resolution.

Calculated $45 \mathrm{keV}$ energy Sodium ion trajectories are shown for the COMPASS tokamak in Fig. 9(a) and (b). These ions can leave the plasma and can be detected far from the separatrix. For the detection we consider a Faraday cup matrix which can be placed close to the plasma, while for ASDEX Upgrade a scintillator-based optical system ${ }^{41}$ has been proposed which needs to be hidden in a port in order to protect it from X-ray and UV radiation. A 5x10 element vacuum compatible and heat resistant Faraday cup matrix has been built using special printed circuit manufacturing technology and tested in the laboratory with a Lithium ion beam between permanent magnets. The calculated ion current distribution on the detector plate, corresponding to trajectories in Fig. 9(a), is shown in Fig. 9(c). The poloidal magnetic field moves this ion cloud approximately horizontally while plasma potential changes would move the cloud vertically due to the ion energy change ${ }^{41}$. These two effects are difficult to separate, therefore modulation of the beam with the beam chopper is considered. The modulation phase distribution in the ion cloud for 500 $\mathrm{kHz}$ modulation frequency is shown in Fig. 9(d). Measuring the phase of the ion current in the Faraday cups would allow separation of magnetic and electric field effects therefore this diagnostic has the potential of resolving both quantities with microsecond-range time resolution.

\section{B. Charge-exchange measurement}

Using the Li-beam as electron donor in charge exchange (CX) diagnostics has been demonstrated on Carbon and Helium ions ${ }^{7-9}$. It has to be noted that the alkali beam density is 1-2 orders of magnitude lower than a typical Hydrogen heating beam density, therefore the signal is also expected to be considerably lower than in the case of heating beam based measurements. The advantage of alkali-beam CX diagostic would be the relaxed geometry constraint for a better spatial resolution due to the smaller beam diameter, measurement possibility in non-NBI heated discharges and better background correction due to more flexible beam modulation. In the above measurements the active CX signal was only a few $10 \%$ of the passive signal, therefore beam modulation was a key element. The measurements were done with spectrometers and CCD cameras which limited the time resolution to a few $100 \mathrm{~ms}$. In the new alkali beam setups described in this paper the beam density is only about 1.5 times higher therefore the situation is expected to be similar. The fast beam chopping would significantly improve background correction possibilties once fast detectors are applied. This could be achieved by a combination of coherence imaging techniques ${ }^{42,43}$ and fast APD/MPPC detectors.

\section{CONCLUSIONS AND OUTLOOK}

Long pulse capable advanced alkali (Li, Na) beam diagnostic setups have been developed. Although the ion source current density is increased only by a factor of 1.5-2, the total extractable charge is higher by an order of magnitude than in previous systems. Combined with a recirculating neutralizer and a long-pulse capable fast light detection setup several ten second long pulses can be measured with $\mathrm{MHz}$ resolution. Using direct imaging onto high efficiency detectors the collected light intensity was increased by 1-2 orders of magnitude enabling density profile and turbulence measurements with twodimensional resolution and microsecond timescale. Several additional possibilities for edge current, ion temperature and flow measurement are under cosideration.

\section{ACKNOWLEDGEMENTS}

This work has been carried out within the framework of the EUROfusion Consortium and has received funding from the Euratom research and training programme 2014-2018 under grant agreement No 633053. The views and opinions expressed herein do not necessarily reflect those of the European Commission. Some technologies have been developed with support of the Hungarian National Development Agency under grant NUKENERGOMFB-00717/718/719/2005. 


\section{REFERENCES}

${ }^{1}$ K. Kadota, K. Tsuchida, Y. Kawasumi, and J. Fujita, Plasma Physics 20, 1011 (1978).

${ }^{2}$ K. McCormick, H. Murmann, and M. El Shaer, Journal of Nuclear Materials 121, 48 (1984).

${ }^{3}$ K. McCormick, Review of Scientific Instruments 56, 1063 (1985). ${ }^{4}$ D. M. Thomas, Review of Scientific Instruments 74, 1541 (2003), http://dx.doi.org/10.1063/1.1526928.

${ }^{5}$ K. Kamiya, T. Fujita, A. Kojima, and H. Kubo, Review of Scientific Instruments 81, 033502 (2010), https://doi.org/10.1063/1.3309793.

${ }^{6}$ A. A. Korotkov, P. D. Morgan, J. Vince, J. Schweinzer, and J. E. Contributors, Review of Scientific Instruments 75, 2590 (2004), https://doi.org/10.1063/1.1763250.

${ }^{7}$ R. P. Schorn, E. Hintz, D. Rusbüldt, F. Aumayr, M. Schneider, E. Unterreiter, and H. Winter, Applied Physics B 52, 71 (1991).

${ }^{8}$ H. Ehmler, J. Baldzuhn, K. McCormick, A. Kreter, T. Klinger, and W.-A. Team, Plasma Physics and Controlled Fusion 45, 53 (2003).

${ }^{9}$ M. Reich, E. Wolfrum, J. Schweinzer, H. Ehmler, L. D. Horton, J. Neuhauser, and A. U. Team, Plasma Physics and Controlled Fusion 46, 797 (2004).

${ }^{10}$ D. M. Thomas, G. R. McKee, K. H. Burrell, F. Levinton, E. L. Foley, and R. K. Fisher, Fusion Science and technology 53, 487 (2008).

${ }^{11}$ E. Wolfrum, F. Aumayr, D. Wutte, H. Winter, E. Hintz, D. Rusbüldt, and R. P. Schorn, Review of Scientific Instruments 64, 2285 (1993), http://dx.doi.org/10.1063/1.1144460.

${ }^{12} \mathrm{~S}$. Zoletnik, S. Fiedler, G. Kocsis, G. K. McCormick, J. Schweinzer, and H. P. Winter, Plasma Physics and Controlled Fusion 40, 1399 (1998).

${ }^{13}$ S. Zoletnik, M. Anton, M. Endler, S. Fiedler, M. Hirsch, K. McCormick, and J. Schweinzer, Physics of Plasmas 6, 4239 (1999), http://dx.doi.org/10.1063/1.873691

${ }^{14}$ M. Willensdorfer, G. Birkenmeier, R. Fischer, F. M. Laggner, E. Wolfrum, G. Veres, F. Aumayr, D. Carralero, L. Guimarais, B. Kurzan, and the ASDEX Upgrade Team, Plasma Physics and Controlled Fusion 56, 025008 (2014).

${ }^{15}$ G. Birkenmeier, F. M. Laggner, M. Willensdorfer, T. Kobayashi, P. Manz, E. Wolfrum, D. Carralero, R. Fischer, B. Sieglin, G. Fuchert, U. Stroth, and the ASDEX upgrade team, Plasma Physics and Controlled Fusion 56, 075019 (2014).

${ }^{16}$ K. McCormick, S. Fiedler, G. Kocsis, J. Schweinzer, and S. Zoletnik, Fusion Engineering and Design 34-35, 125 (1997).

${ }^{17}$ P. K. Roy, W. G. Greenway, and J. W. Kwan, Review of Scientific Instruments 83, 043303 (2012), https://doi.org/10.1063/1.4704457.

${ }^{18}$ D. M. Thomas, Review of Scientific Instruments 66, 806 (1995), http://dx.doi.org/10.1063/1.1146227.

${ }^{19}$ A. Kojima, K. Kamiya, H. Iguchi, T. Fujita, H. Kakiuchi, and Y. Kamada, Review of Scientific Instruments 79, 093502 (2008), https://aip.scitation.org/doi/pdf/10.1063/1.2964225.

${ }^{20}$ M. Brix, D. Dodt, A. Korotkov, P. Morgan, D. Dunai, R. Fischer, A. Meigs, I. S. Nedzelskiy, J. Schweinzer, J. Vince, and S. Zoletnik, Review of Scientific Instruments 81, 10D733 (2010), http://dx.doi.org/10.1063/1.3502320.

${ }^{21}$ M. Lampert, G. Anda, A. Czopf, G. Erdei, D. Guszejnov, A. Kovácsik, G. I. Pokol, D. Réfy, Y. U. Nam, and S. Zoletnik, Review of Scientific Instruments 86, 073501 (2015), http://dx.doi.org/10.1063/1.4923251.

${ }^{22}$ S. Zoletnik, G. H. Hu, B. Tl, D. Dunai, G. Anda, O. Asztalos, G. I. Pokol, S. Klvin, J. Nmeth, and T. Krizsanczi, Review of Scientific Instruments 89, 063503 (2018), https://doi.org/10.1063/1.5017224.

${ }^{23}$ G. Anda, G. Petravich, S. Zoletnik, and S. Bató, Fusion Engineering and Design 74, 715 (2005).

${ }^{24}$ G. Anda, D. Dunai, M. Lampert, T. Krizsanczi, J. Nmeth, S. Bat, Y. U. Nam, G. H. Hu, and S. Zoletnik,
Review of Scientific Instruments 89, $013503 \quad$ (2018), https://doi.org/10.1063/1.5004126.

${ }^{25}$ M. Willensdorfer, E. Wolfrum, R. Fischer, J. Schweinzer, M. Sertoli, B. Sieglin, G. Veres, and F. Aumayr, Review of Scientific Instruments 83, 023501 (2012), http://dx.doi.org/10.1063/1.3682003.

${ }^{26}$ S. Zoletnik, G. Petravich, A. Bencze, M. Berta, S. Fiedler, K. McCormick, and J. Schweinzer, Review of Scientific Instruments $\mathbf{7 6}$, 073504 (2005).

${ }^{27}$ D. Dunai, S. Zoletnik, J. Sárközi, and A. R. Field, Review of Scientific Instruments 81, 103503 (2010), https://doi.org/10.1063/1.3488458.

${ }^{28}$ S. Zoletnik, L. Bardoczi, A. Krämer-Flecken, Y. Xu, I. Shesterikov, S. Soldatov, G. Anda, D. Dunai, G. Petravich, and the TEXTOR Team, Plasma Physics and Controlled Fusion 54, 065007 (2012).

${ }^{29}$ G. Anda, A. Bencze, M. Berta, D. Dunai, P. Hacek, J. Krbec, D. Réfy, T. Krizsanoczi, S. Bató, T. Ilkei, I. Kiss, G. Veres, and S. Zoletnik, Fusion Engineering and Design 108, 1 (2016).

${ }^{30}$ M. Berta, G. Anda, A. Bencze, D. Dunai, P. Hacek, M. Hron, A. Kovácsik, J. Krbec, R. Pánek, D. Réfy, G. Veres, V. Weinzettl, and S. Zoletnik, Fusion Engineering and Design 96-97, 795 (2015), proceedings of the 28th Symposium On Fusion Technology (SOFT-28).

${ }^{31}$ O. Grover, J. Seidl, D. Réfy, J. Adamek, P. Vondracek, M. Tomes, P. Junek, P. Hacek, J. Krbec, V. Weinzettl, M. Hron, and S. Zoletnik, accepted for publication in Nuclear Fusion (2018).

${ }^{32}$ D. I. Réfy, M. Brix, R. Gomes, B. Tál, S. Zoletnik, D. Dunai, G. Kocsis, S. Kálvin, T. Szabolics, and J. Contributors, Review of Scientific Instruments (Accepted) (2018).

${ }^{33}$ D. Guszejnov, G. I. Pokol, I. Pusztai, D. Refy, S. Zoletnik, M. Lampert, and Y. U. Nam, Review of Scientific Instruments 83, 113501 (2012), http://dx.doi.org/10.1063/1.4764564.

${ }^{34}$ R. Fischer, E. Wolfrum, J. Schweinzer, and the ASDEX Upgrade Team, Plasma Physics and Controlled Fusion 50, 085009 (2008).

${ }^{35}$ E. Wolfrum, J. Schweinzer, D. Bridi, K. Igenbergs, J. Kamleitner, and F. Aumayr, Journal of Nuclear Materials 390-391, 1110 (2009), proceedings of the 18th International Conference on Plasma-Surface Interactions in Controlled Fusion Device.

${ }^{36}$ Fusion Instruments Kft., "APDCAM Setup Considerations for Optimal Signal to Noise Ratio" (2016).

${ }^{37}$ A. R. Field, D. Dunai, R. Gaffka, Y. c. Ghim, I. Kiss, B. Mészáros, T. Krizsanóczi, S. Shibaev, and S. Zoletnik, Review of Scientific Instruments 83, 013508 (2012), http://dx.doi.org/10.1063/1.3669756.

${ }^{38} \mathrm{~J}$. Gonzalez-Martin, J. Ayllon-Guerola, M. Garcia-Munoz, A. Herrmann, P. Leitenstern, P. Popken, W. De Marne, S. Zoletnik, A. Kovácsik, J. Galdon-Quiroga, J. Rivero-Rodriguez, M. Rodriguez-Ramos, L. Sanchis-Sanchez, J. DominguezAbascal, A. U. Team, and M. Team, This conference (2018).

${ }^{39}$ D. M. Thomas, A. W. Leonard, L. L. Lao, T. H. Osborne, H. W. Mueller, and D. F. Finkenthal, Phys. Rev. Lett. 93, 065003 (2004).

${ }^{40}$ M. Berta, G. Anda, M. Aradi, A. Bencze, C. Buday, I. Kiss, S. Tulipán, G. Veres, S. Zoletnik, J. Havlcek, and P. Hacek, Fusion Engineering and Design 88, 2875 (2013).

${ }^{41}$ J. Galdon-Quiroga, J. Rivero-Rodriguez, M. Garcia-Munoz, G. Birkenmeier, E. Viezzer, J. Ayllon-Guerola, M. Dunne, J. Garcia-Lopez, J. Gonzalez-Martin, M. C. Jimenez-Ramos, M. Rodriguez-Ramos, L. Sanchis-Sanchez, E. Wolfrum, and A. U. Team, Journal of Instrumentation 12, C08023 (2017).

${ }^{42} \mathrm{~J}$. Howard, Journal of Physics B: Atomic, Molecular and Optical Physics 43, 144010 (2010).

${ }^{43}$ J. Chung, R. König, J. Howard, M. Otte, and T. Klinger, Plasma Physics and Controlled Fusion 47, 919 (2005). 